\title{
Characterization of highly proliferative decidual precursor cells during the window of implantation in human endometrium
}

\author{
Maria Diniz-da-Costa $^{1,2}$ ～Chow-Seng Kong ${ }^{1}$ ～Katherine J. Fishwick ${ }^{1}$ | \\ Thomas Rawlings $^{1}$ | Paul J. Brighton ${ }^{1}$ | Amelia Hawkes ${ }^{2}$ | Joshua Odendaal ${ }^{2}$ | \\ Siobhan Quenby ${ }^{1,2,3}$ | Sascha Ott ${ }^{1,2,3}$ | Emma S. Lucas ${ }^{1,3}$ | Pavle Vrljicak ${ }^{1}$ | \\ Jan J. Brosens ${ }^{1,2,3}$ []
}

${ }^{1}$ Division of Biomedical Sciences, Warwick Medical School, University of Warwick,

Coventry, UK

${ }^{2}$ Tommy's National Centre for Miscarriage Research, University Hospitals Coventry and Warwickshire National Health Service Trust, Coventry, UK

${ }^{3}$ Centre for Early Life, University of Warwick, Coventry, UK

\section{Correspondence}

Jan J. Brosens, MD, PhD, Division of Biomedical Sciences, Warwick Medical School, University of Warwick, Coventry CV2 2DX, UK.

Email: j.j.brosens@warwick.ac.uk

Funding information

Wellcome Trust Investigator Award, Grant/ Award Number: 212233/Z/18/Z; Tommy's

National Miscarriage Research Centre

\begin{abstract}
Pregnancy depends on the wholesale transformation of the endometrium, a process driven by differentiation of endometrial stromal cells (EnSC) into specialist decidual cells. Upon embryo implantation, decidual cells impart the tissue plasticity needed to accommodate a rapidly growing conceptus and invading placenta, although the underlying mechanisms are unclear. Here we characterize a discrete population of highly proliferative mesenchymal cells (hPMC) in midluteal human endometrium, coinciding with the window of embryo implantation. Single-cell transcriptomics demonstrated that hPMC express genes involved in chemotaxis and vascular transmigration. Although distinct from resident EnSC, hPMC also express genes encoding pivotal decidual transcription factors and markers, most prominently prolactin. We further show that hPMC are enriched around spiral arterioles, scattered throughout the stroma, and occasionally present in glandular and luminal epithelium. The abundance of hPMC correlated with the in vitro colony-forming unit activity of midluteal endometrium and, conversely, clonogenic cells in culture express a gene signature partially conserved in hPMC. Cross-referencing of single-cell RNA-sequencing data sets indicated that hPMC differentiate into a recently discovered decidual subpopulation in early pregnancy. Finally, we demonstrate that recurrent pregnancy loss is associated with hPMC depletion. Collectively, our findings characterize midluteal hPMC as novel decidual precursors that are likely derived from circulating bone marrow-derived mesenchymal stem/stromal cells and integral to decidual plasticity in pregnancy.
\end{abstract}

\section{KEYWORDS}

bone-marrow-derived stem cells, decidualization, embryo implantation, endometrium, mesenchymal stem cells, miscarriage, pregnancy 


\section{1 | INTRODUCTION}

The uterine mucosa-the endometrium-is a highly regenerative tissue capable of adopting different physiological states during the reproductive years. $^{1,2}$ In the midluteal phase of the menstrual cycle, the endometrium starts remodeling intensively in response to elevated progesterone levels and rising intracellular cyclic AMP levels, ${ }^{3}$ heralding the start of a short window during which an embryo can implant. ${ }^{4}$ This process, termed decidualization, begins with an acute stress response in endometrial stromal cells (EnSC), ${ }^{5}$ resulting in cell cycle arrest, release of proinflammatory mediators, and influx of uterine natural killer (uNK) cells. ${ }^{6,7}$ After a lag period of approximately 4 days, phenotypic decidual cells emerge, coinciding with the closure of the implantation window. ${ }^{6-8}$ Decidual cells are progesterone-dependent secretory cells with a well-developed endoplasmic reticulum and enlarged nuclei. They are refractory to oxidative and metabolic stress signals, ${ }^{9-12}$ and form a quasi-autonomous, antiinflammatory matrix around the implanting embryo, which enables invading extravillous trophoblast to form a hemochorial placenta. ${ }^{3}$ In addition, the maternal decidua creates a tolerogenic microenvironment that harbors an abundance of infiltrating immune cells, foremost uNK cells but also macrophages, dendritic cells, and regulatory T cells. ${ }^{13}$

Recently, we provided evidence that inflammatory reprogramming of EnSC also leads to the emergence of senescent decidual cells. ${ }^{6,8,14}$ Unlike decidual cells, senescent decidual cells are progesterone resistant. $^{8}$ They secrete a complex mixture of extracellular matrix (ECM) proteins and proteinases, proinflammatory cytokines, and chemokines, designated senescence-associated secretory phenotype (SASP), which causes sterile inflammation and induces secondary senescence in neighboring decidual cells. ${ }^{6,8}$ Hence, in a non-conception cycle, falling progesterone levels during the late-luteal phase result in a preponderance of senescent decidual cells, an influx of neutrophils, ECM breakdown, and menstrual shedding of the superficial endometrial layer. ${ }^{15}$ By contrast, continuous progesterone signaling upon successful embryo implantation enables decidual cells to engage and activate uNK cells, which in turn target and eliminate senescent decidual cells through perforin- and granzyme-containing granule exocytosis., ${ }^{6,8}$ Perturbations that lead to an excessive or persistent pro-senescent decidual response presents the embryo with a uterine mucosa that is easy to invade but also prone to breakdown. Clinically, a pro-senescent decidual response is associated with rapid conceptions (ie, "super-fertility") and recurrent pregnancy loss. $^{8,16,17}$

Despite these emerging insights, it remains unclear how the maternal decidua acquires the necessary plasticity to accommodate rapid expansion in early gestation. Studies in mice have provided compelling evidence that pregnancy is associated with engraftment of circulating bone marrowderived mesenchymal stem/stromal cells (BDMSC) into the decidua, ${ }^{18-20}$ which differentiate into nonhematopoietic prolactin-expressing decidual cells. ${ }^{20}$ A recent study demonstrated that bone marrow transplants from wild-type mice to mice carrying a heterozygous deletion of Hoxa11, a pivotal decidual transcription factor, not only restore the decidual response but also prevent pregnancy loss in these animals. ${ }^{20}$ This observation both underscores the importance of BDMSC in establishing a robust placentaldecidual interface and signposts a novel strategy for the prevention of

\section{Significance statement}

Transformation of cycling endometrium into the decidua of pregnancy requires extensive tissue remodeling. Perturbations in this process lead to breakdown of the maternal-fetal interface and miscarriage. Here the authors report the characterization of decidual precursor cells during the window of implantation. The authors demonstrate that decidual precursors are clonogenic and primed for exponential growth. They likely originate from bone marrow-derived mesenchymal stem/stromal cells and give rise to a distinct decidual subpopulation in pregnancy. Recurrent pregnancy loss is associated with loss of decidual precursor cells prior to conception, raising the possibility that they can be harnessed for the prevention of pregnancy disorders, including miscarriages and preterm labor.

intractable pregnancy disorders, such as recurrent pregnancy loss and preterm birth. Whether BDMSC contribute to the decidual transformation of human endometrium is, however, not known.

Based on single-cell transcriptomics, we recently described the presence of a discrete population of highly proliferative mesenchymal cells (hPMC) in midluteal endometrium. ${ }^{8}$ Here, we report on the characterization of these cells. Our findings indicate that hPMC are the human equivalent of bone marrow-derived decidual precursor cells in mice. We provide evidence that hPMC impart clonogenicity of midluteal endometrium and give rise to a distinct subpopulation of decidual cells at the maternal-fetal interface in pregnancy. We further demonstrate that a lack of these novel human decidual precursor cells is associated with recurrent pregnancy loss.

\section{2 | MATERIALS AND METHODS}

\subsection{Endometrial sample collection}

The collection of endometrial biopsies was approved by the NHS National Research Ethics-Hammersmith and Queen Charlotte's \& Chelsea Research Ethics Committee (REC reference: 1997/5065) and Tommy's National Reproductive Health Biobank (REC reference: 18/WA/0356). Samples were obtained using a Wallach Endocell sampler 5 to 10 days after the pre-ovulatory luteinizing hormone (LH) surge. Demographic details of the tissue samples used in different experiments are shown in Table S1.

\section{2 | Endometrial tissue processing, primary cell culture, and CFU assay}

Processing of endometrial biopsies, isolation of sushi domain containing 2 (SUSD2)-positive cells using magnetic-activated cell sorting, 
and culturing of primary endometrial cells were carried out as described in our published step-by-step protocol. ${ }^{21} \mathrm{CFU}$ assays were also performed as described previously. ${ }^{22}$ Briefly, EnSC were seeded at a clonal density of 30 cells $/ \mathrm{cm}^{2}$ on $1 \mathrm{mg} / \mathrm{mL}$ fibronectin-coated 6-well plates and cultured in 10\% DMEM/F12 medium supplemented with basic fibroblast growth factor (bFGF; $10 \mathrm{ng} / \mathrm{mL}$; Merck Millipore, Watford, UK) for 10 days with a $50 \%$ media change on day 7. Colonies were monitored microscopically to ensure that they were derived from single cells. After 10 days, colonies were washed with PBS and fixed in $4 \%$ formaldehyde for 10 minutes at room temperature (RT) before staining with hematoxylin for 4 minutes. Colonies were visualized on EVOS FL AUTO microscope (Life Technologies, Paisley, UK). Clusters of $\geq 50$ cells were counted and cloning efficiency (\%) was calculated as the number of colonies formed/number of cells seeded $\times 100$.

\section{3 | Immunohistochemistry}

Endometrial biopsies were fixed overnight in $10 \%$ neutral buffered formalin at $4{ }^{\circ} \mathrm{C}$ and embedded in Surgipath Formula "R" paraffin using the Shandon Excelsior ES Tissue processor (Thermo Fisher Scientific). Tissues were sliced into $3 \mu \mathrm{M}$ sections on a microtome and adhered to coverslips by overnight incubation at $60^{\circ} \mathrm{C}$. Deparaffinization, antigen retrieval, antibody staining, hematoxylin counter stain and DAB color development were fully automated in a Leica BondMax autostainer (Leica BioSystems). Tissue sections were labeled for anillin (AMAB90660, Sigma) using a 1:100 dilution. Stained slides were dehydrated, cleared and cover-slipped in a Tissue-Tek Prisma Automated Slide Stainer, model 6134 (Sakura Flinetek, Inc, California) using DPX coverslip mountant. Bright-field images were obtained on a Mirax Midi slide scanner with a $20 x$ objective lens, and three randomly selected areas of interest underlying the luminal epithelium were captured using Panoramic Viewer v1.15.3 (3DHISTECH Ltd, Budapest, Hungary). Each field was divided manually into three compartments: stroma, glandular epithelium, and luminal epithelium. ImageJ image analysis software (Rasband, W. S., ImageJ, National Institutes of Health) was used to quantify anillin ${ }^{+}$cells with staining intensity manually determined by background thresholding. The percentage of anillin ${ }^{+}$cells was calculated (ie, anillin ${ }^{+}$cells/total cells $\times 100$ ) in each field of view and for each cellular compartment.

\subsection{Immunofluorescence microscopy}

CFUs and EnSC monolayers in 6-well plates were fixed in 4\% paraformaldehyde, permeabilized with 0.5\% Triton X-100 (Sigma Aldrich) and blocked with $1 \%$ BSA/PBS. Cells were probed with mouse anti-anillin monoclonal primary antibody (1:100, Sigma-Aldrich) overnight at $4^{\circ} \mathrm{C}$. Labeled cells were stained with Alexa-Fluor 594 anti-mouse secondary antibody (1:1000, Invitrogen) for 1 hour at RT and counterstained with VECTASHIELD antifade mounting medium with DAPI (Vector Laboratories, Peterborough, UK). Images were captured on an EVOS
FL AUTO fluorescence microscope. Anillin ${ }^{+}$cells were quantified in three randomly selected fields relative to total number of DAPIstained cells using ImageJ image analysis software. Tissue sections were double labeled overnight at $4^{\circ} \mathrm{C}$ with antibodies against anillin (mouse or rabbit) and either CD34 (1:250, Abcam, Cambridge, UK), CD56 (1:200, Leica Biosystems), or CD163 (1:200, Abcam). Labeled cells were stained with Alexa Fluor 594 anti-mouse secondary antibody and Alexa Fluor 488 anti-rabbit secondary antibody. Tissue autofluorescence was eliminated using the TrueVIEW autofluorescence quenching kit (Vector Laboratories). Tissue sections were stained with ProLong Gold antifade mounting medium with DAPI (Invitrogen) and examined using an EVOS FL Auto fluorescence microscope (Life Technologies).

\section{5 | RNA-sequencing and analysis}

Total RNA was extracted from CFUs and standard cultures using the AllPrep DNA/RNA Micro Kit (Qiagen, Manchester, UK) following the manufacturer's protocol. RNA concentration was assessed using the Qubit RNA BR assay and RNA quality was analyzed on an Agilent 2100 Bioanalyzer (Agilent Technologies). Libraries were prepared using the TruSeq RNA Library preparation kit V2 (Illumina, Cambridge, UK) and sequenced on HiSeq 4000 (Illumina) with 75 bp paired-end reads at the Wellcome Trust Centre for Human Genetics (Oxford, UK). Transcriptomic maps of paired-end reads were generated using Bowtie-2.2.3, Samtools-0.1.19, and Tophat-2.0.12 against the hg19 reference transcriptome. Transcript counts were assessed by HTSeq0.6.1 using the reverse strand setting and intersection nonempty mode and counts were assigned to Ensembl gene IDs. Differential gene expression was evaluated with the DESeq2 package v1.28.1 in R, using the Wald test and Benjamini and Hochberg FDR corrected $P<0.05$. Principal component analysis on the log2-normalized count data was performed in MATLAB R2020b. RNA-seq library were analyzed using Euclidean distance were plotted with pheatmap package v1.0.12 in R. RNA-seq data were deposited in the Gene Expression Omnibus (http://www.ncbi.nlm.nih.gov/geo/), accession number GSE159266.

\subsection{Single-cell transcriptome analyses}

Analysis of previously reported single-cell RNA-seq data of midluteal endometrial biopsies (GSE127918) was performed with Seurat R package v3.2.2. To minimize artifacts, only cells with between 200 and 6000 features and less than $5 \%$ mitochondrial gene content were included in our analysis. Single cells were assigned to phases of the cell cycle using the CellCycleScoring function using validated cell cycle marker genes. ${ }^{23}$ Following scaling and regression of the cell cycle information, the first 10 principal components were used to find clusters using a resolution of 0.4 . Marker genes were obtained with the FindMarkers function with Bonferroni correction. Heatmaps of gene expression were generated with the gplots package v3.1.0. Ligandreceptor analyses were performed with CellphoneDB (www. CellPhoneDB.org). ${ }^{24}$ Single cell transcriptome data of the maternal-fetal 
(A)
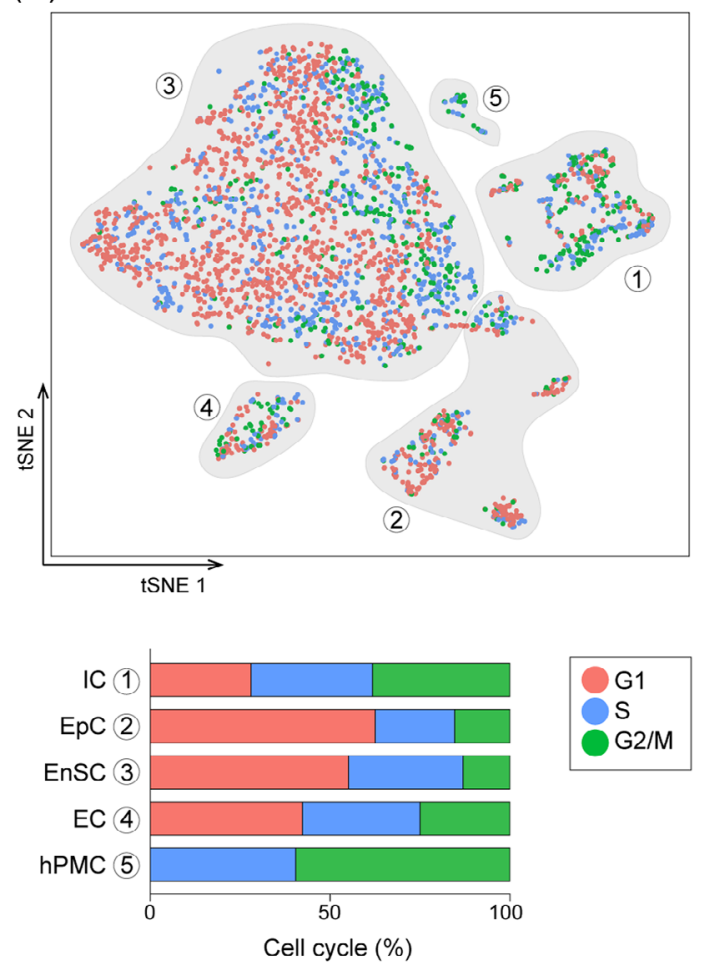

(D)

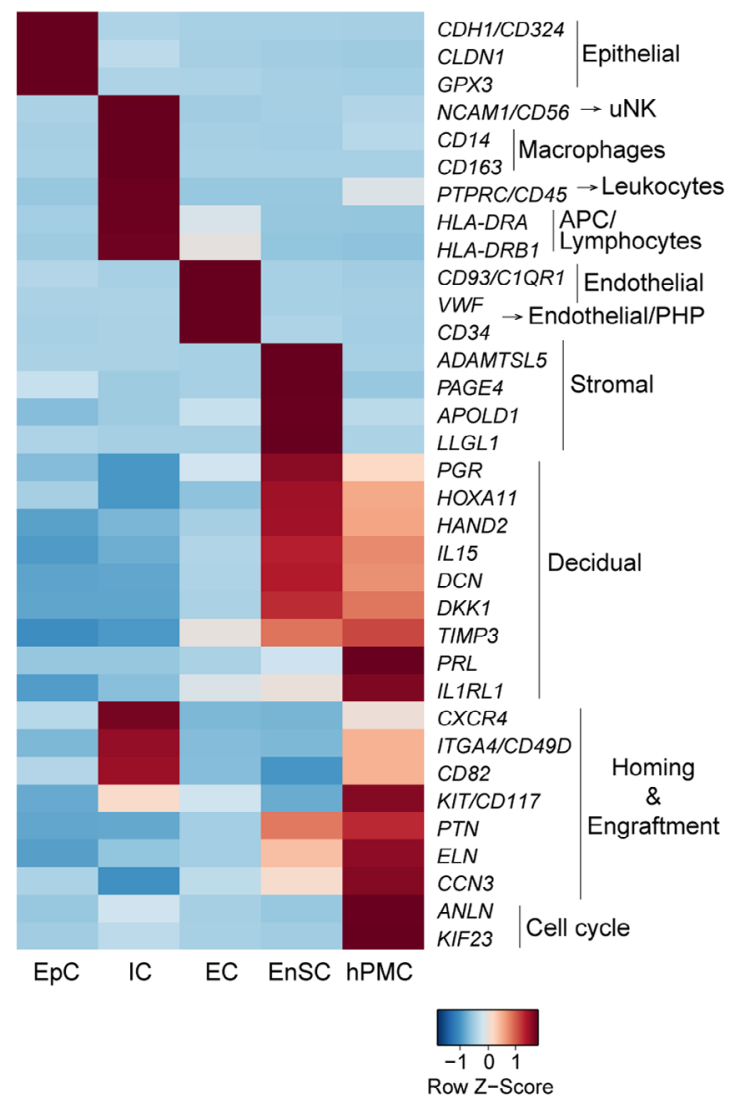

(B)

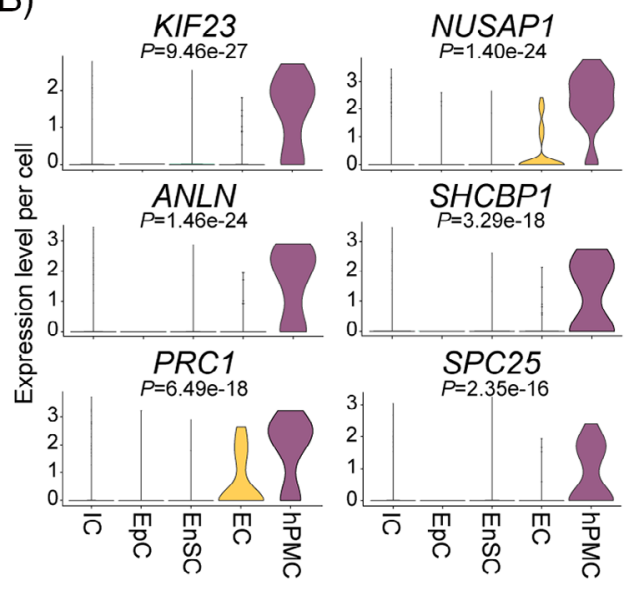

(C)

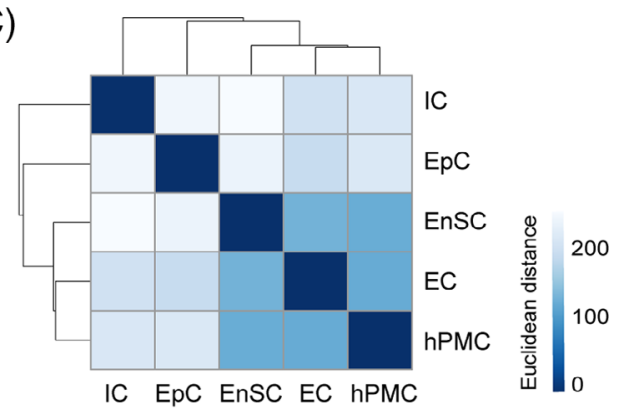

(E)

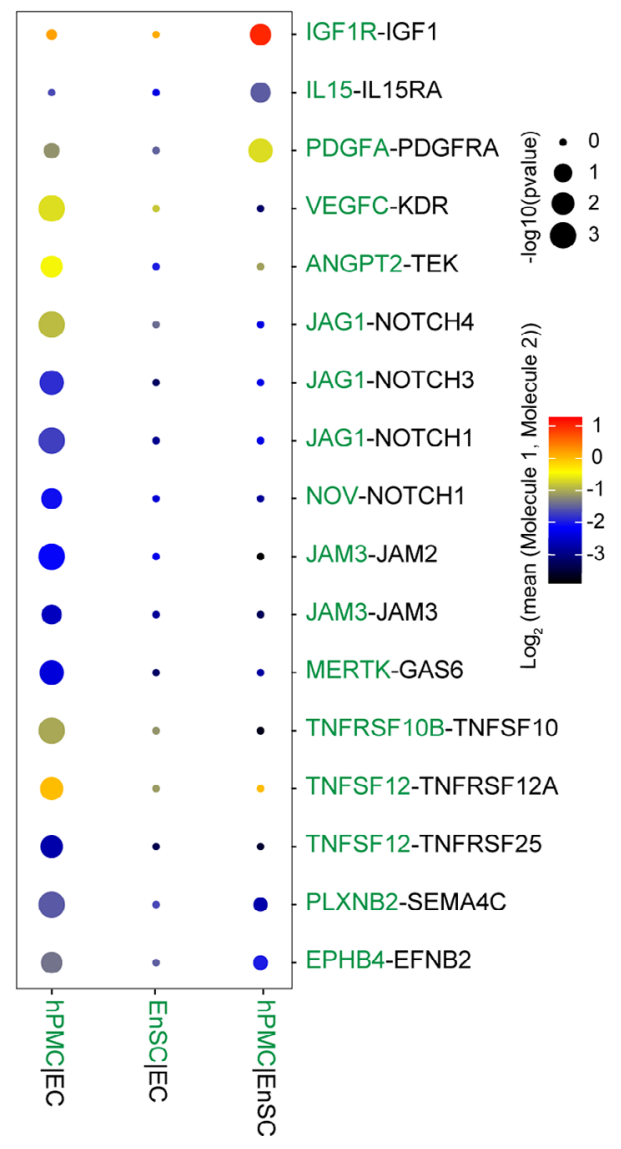

FIGURE 1 Legend on next page. 
interface were obtained from ArrayExpress (E-MTAB-6701). Cell cycle phases were assigned as described above, but not regressed. Differential gene expression analysis was performed using the published cell labels. Informative genes were visualized using an on-line browsing tool (https://maternal-fetal-interface.cellgeni.sanger.ac.uk/).

\subsection{Statistical analysis}

Data analyses were performed using the statistical package Graphpad Prism 6 and R v4.0.2. Mann-Whitney $U$ test, Student $t$-test, and oneway ANOVA on ranks (Kruskal-Wallis) test were used as appropriate. Spearman rank-order correlation coefficient was calculated to measure the strength and direction of association between two variables. $P<.05$ was considered statistically significant.

\section{$3 \mid$ RESULTS}

\section{1 | Characterization of hPMC in midluteal endometrium}

We recently reported on single-cell RNA-sequencing analysis of midluteal endometrial biopsies, that is, coinciding with the window of implantation. ${ }^{8}$ A notable observation was the presence of a discrete cell population comprising $1.8 \%$ of all nonimmune stromal cells. This population was designated "highly proliferating mesenchymal cells" (hPMC) based on 127 upregulated genes (FDR corrected $P<.05$ ) involved in cell cycle progression. In agreement, gene ontology analysis yielded terms such as "cell cycle" (FDR corrected $P=6.3 \times 10^{-66}$ ), "cell division" (FDR corrected $P=4.77 \times 10^{-41}$ ), and "regulation of mitotic cell cycle" (FDR corrected $P=1.41 \times 10^{-22}$ ). To explore the nature of hPMC, we first computationally determined the cell cycle status of all cells in midluteal endometrium (Figure $1 \mathrm{~A}$ ). The hPMC population consisted exclusively of cells in either S-phase $(40 \%)$ or G2/M-phase (60\%) (Figure 1A). By contrast, the majority of EnSC and endometrial epithelial cells (EpC) were assigned to G1 (55\% and 63\%, respectively). The abundance of endothelial cells (EC) and immune cells (IC) in S or $\mathrm{G} 2 / \mathrm{M}$ phase was $58 \%$ and $72 \%$, respectively (Figure $1 \mathrm{~A}$ ). Next, we compared gene expression in hPMC assigned to the $G 2 / M$ phase to all other cells in $G 2 / M$ across different populations. This analysis yielded 19 differentially expressed cell cycle genes (FDR corrected $P<.05$ ), all markedly upregulated in
hPMC (Figure 1B and Table S2). To determine the provenance of hPMC, we constructed a correlation matrix heatmap and dendrogram to show hierarchical clustering of different endometrial cell populations (Figure 1C). The heatmap depicts the Euclidian distance in gene expression between cell populations whereas the distance between the dendrogram branches reflects clustering according to the complete linkage method. hPMC clustered with EC and EnSC, but not with $\mathrm{EpC}$ or IC, suggesting shared provenance (Figure 1C).

Mining of gene expression data showed that hPMC do not express genes that encode for canonical IC, EC, or EpC markers (Figure 1D). They also do not express several EnSC-specific genes that are constitutively expressed across the menstrual cycle (eg, ADAMTSL5, PAGE4, APOLD1, and LLGL1) (Figure 1D and Figure S1). These EnSC-specific marker genes are abundantly expressed, rendering it unlikely that lack of expression in hPMC is an erroneous observation attributable to relative enrichment of cell cycle genes in this subpopulation. Furthermore, hPMC share with EnSC the expression of multiple decidual genes, including DCN (coding decorin), DKK1 (dickkopf WNT signaling pathway inhibitor 1), IL15 (interleukin 15), and TIMP3 (TIMP metallopeptidase inhibitor 3). $P R L$ (prolactin), a widely used marker gene of decidualizing EnSC in vitro, ${ }^{3}$ is conspicuously enriched in hPMC in vivo. Another notably enriched decidual gene is IL1RL1, which codes both the transmembrane and soluble IL-33 receptors (termed ST2L and sST2, respectively). ${ }^{7}$ hPMC also express key decidual transcription factors, including $P G R$ (coding the progesterone receptor), ${ }^{25}$ HAND2 (heart and neural crest derivatives expressed 2 ), ${ }^{26}$ and HOXA11 (homeobox A11), ${ }^{27}$ indicating commitment to decidual differentiation (Figure 1D). On the other hand, hPMC share with IC genes involved in chemotaxis (eg, CXCR4 and KIT) and transendothelial migration (eg, ITGA4 and CD82). ${ }^{28}$ However, unlike IC, hPMC also express genes coding for secreted factors implicating in MSC self-renewal and engraftment (Figure 1D), including tropoelastin $(E L N),{ }^{29}$ pleiotrophin $(P T N),{ }^{30}$ and cellular communication network factor 3 (CCN3). ${ }^{31} \mathrm{Next}$, we used CellPhoneDB to explore putative interactions between hPMC, EC and EnSC. This computational tool takes into account the subunit architecture of both ligands and receptors in heteromeric complexes. ${ }^{13,24}$ As shown in Figure $1 \mathrm{E}$ and Table S3, CellPhoneDB predicted many more enriched receptor-ligand interactions between EC and hPMC than with EnSC. Specifically, hPMC selectively encode ligands that bind various EC receptors, including NOTCH 1, 3 and 4, TIE2 receptor (TEK), and VEGFR (KDR). On the other hand, activation of

FIGURE 1 Transcriptomic analysis of hPMC. A, Top panel shows t-SNE projection of mid-secretory endometrial cell populations [epithelial cells (EpC), immune cells (IC), endothelial cells (EC), stromal cells (EnSC) and highly proliferative mesenchymal cells (hPMC)] color-coded to indicate cells in different phases of the cell cycle (G1, $S$ and G2/M). Lower panel shows the proportion of cells in the different phases of the cell cycle for each population. B, Violin plots showing the relative expression in G2/M phase of six genes highly enriched in hPMC. FDR corrected $P$-values are also shown. C, Heatmap showing Euclidean distance between different cell populations based on average expression values. $\mathrm{D}$, Heatmap showing relative expression (z-score) of genes used to characterize hPMC. Red and blue represent high and low gene expression, respectively, as indicated by the color key. E, Overview of selected ligand-receptor interactions generated by Cellphone DB tool. FDR corrected $P$-values are reflected by circle sizes. The means of the average expression level of interacting molecule 1 in cluster 1 and interacting molecule 2 in cluster 2 are indicated by the color key 
insulin-like growth factor 1 receptor (IGF1R) is predicted to play a role in chemotaxis of hPMC within the stromal compartment (Figure 1E). Intriguingly, a recent study reported activation of IGF1R by CXCL14, ${ }^{32}$ an orphan chemokine abundantly expressed in midluteal endometrium. ${ }^{33}$ Taken together, the data suggest that hPMC are trans-endothelial migratory cells committed to decidual differentiation.
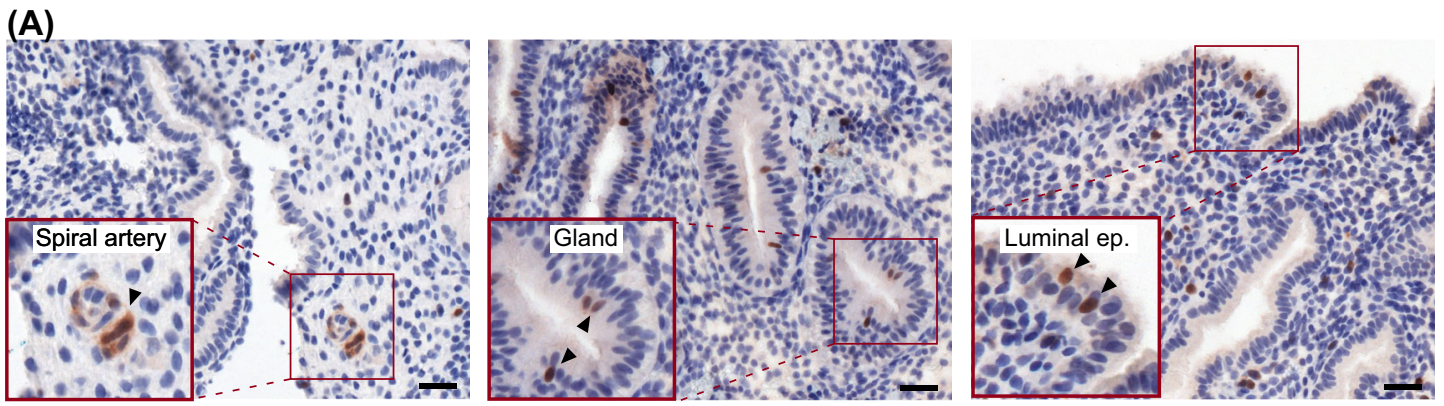

(B)

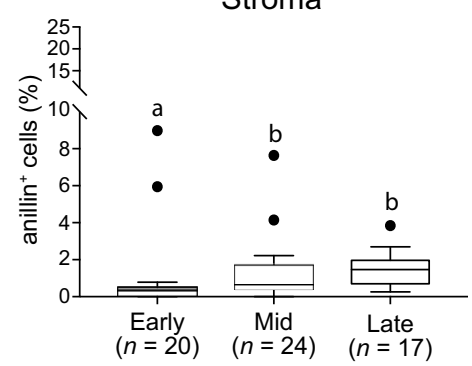

(C)

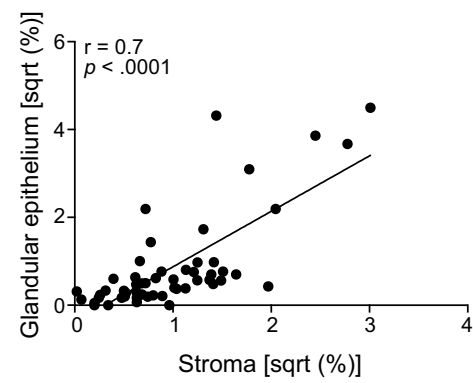

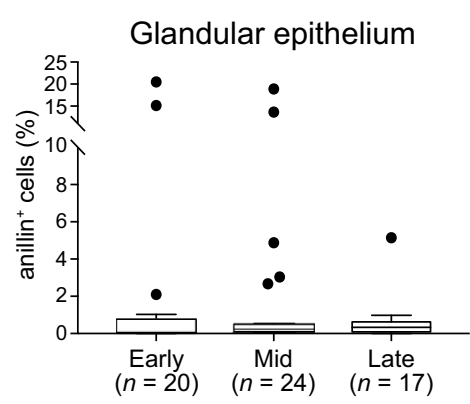
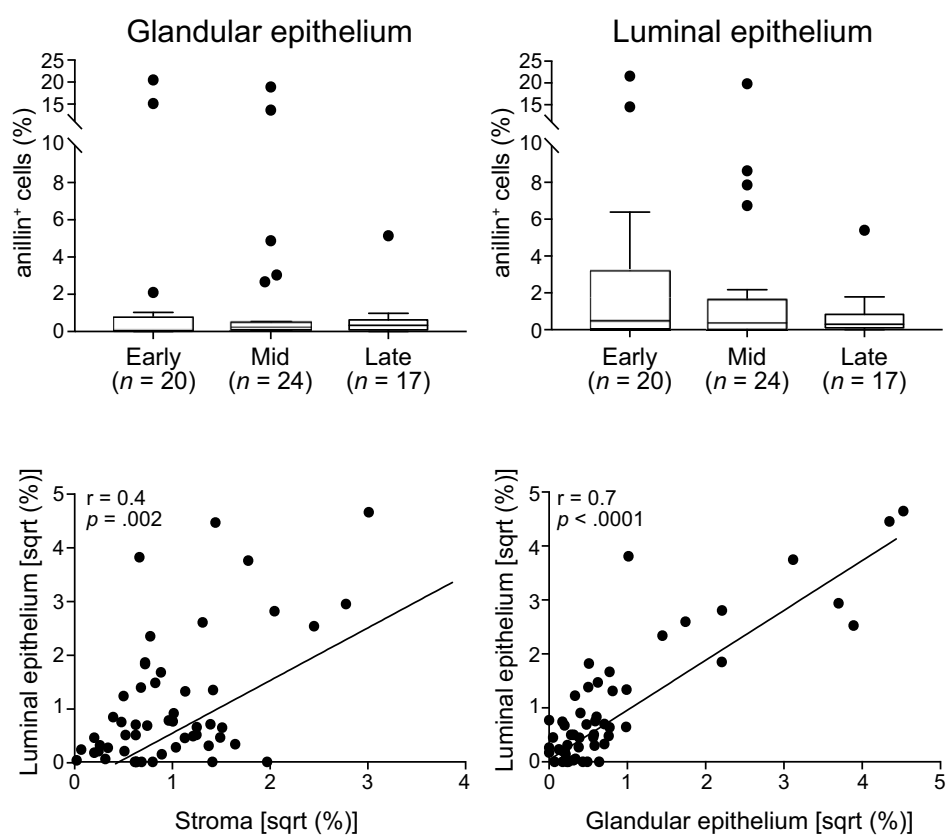

(E)
(D)

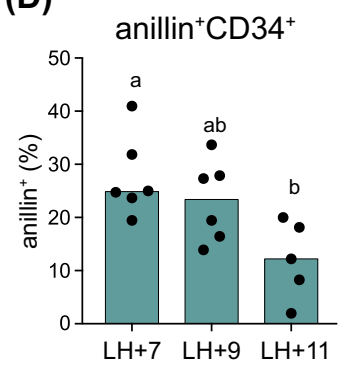

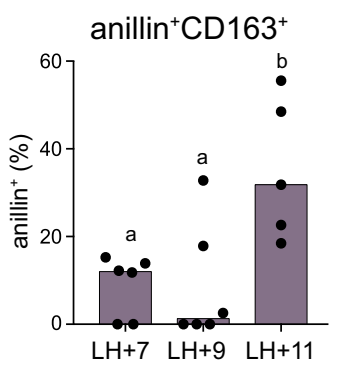

anillin ${ }^{+} \mathrm{CD} 56^{+}$

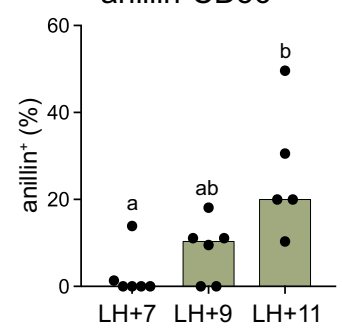

FIGURE 2 Spatiotemporal distribution of anillin ${ }^{+}$cells in peri-implantation endometrium. A, Examples of anillin ${ }^{+}$cells in endometrial stroma and glandular and luminal epithelium. Scale bar $=20 \mu \mathrm{m}$. B, Quantification of anillin ${ }^{+}$cells in different endometrial compartments during the early$(\mathrm{LH}+5 / 6)$, mid- $(\mathrm{LH}+7 / 9)$, and late-secretory $(\mathrm{LH}+10 / 11)$ phase of the cycle. The number of biopsies analyzed at each timepoint is indicated. Different letters above box plots indicate that groups are significantly different from each other at $P<.05$ (one-way ANOVA on ranks test). C, Spearman's rank correlation of anillin ${ }^{+}$cells in endometrial stroma, glandular epithelium and luminal epithelium. D, Quantification of anillin ${ }^{+} \mathrm{CD} 34^{+}$ hematopoietic/endothelial precursor cells, anillin ${ }^{+} \mathrm{CD} 163^{+}$macrophages, and anillin ${ }^{+} \mathrm{CD} 56^{+} \mathrm{uNK}$ cells in peri-implantation endometrium. Different letters above the box plots indicate that groups are significantly different from each other at $P<.05$ (one-way ANOVA on ranks test). E, Relative proportions of anillin ${ }^{+} \mathrm{IC}$ and $\mathrm{hPMC}$ (nonimmune anillin ${ }^{+}$cells) during the midluteal window of implantation (LH $+7 / 9$ ) and late-secretory phase $(\mathrm{LH}+11)$ 


\subsection{Spatiotemporal distribution of hPMC in luteal phase endometrium}

Cross-referencing of potential hPMC markers with the Human Protein Atlas (http://www.proteinatlas.org) revealed the presence of rare anillin ${ }^{+}$ cells scattered throughout the endometrium. ${ }^{34}$ Anillin, encoded by ANLN, is an actin-binding protein involved in cytokinesis, cell growth and migration. ${ }^{35}$ To examine if anillin could serve as a marker for hPMC, immunohistochemistry was performed on 61 LH-timed endometrial biopsies obtained across the luteal phase. This analysis confirmed that anillin ${ }^{+}$cells are rare and dispersed throughout the stroma, although frequently enriched around the terminal spiral arterioles (Figure 2A). Unexpectedly, some anillin ${ }^{+}$cells were also found in glandular and luminal epithelium (Figure 2A). The average abundance of anillin ${ }^{+}$cells [median (interquartile range, $I Q R)]$ in the stromal compartment across the luteal phase was 0.6 (1.3)\% vs $0.4(0.7) \%$ and $0.2(0.6) \%$ in the luminal and glandular epithelium, respectively. In contrast to glandular or luminal epithelium, anillin ${ }^{+}$cells increased significantly in the stroma upon progression from early to latesecretory phase of the cycle (Figure 2B). Quantification of anillin ${ }^{+}$cells showed a strong correlation between the three main cellular compartments (Figure $2 \mathrm{C}$ ), especially between the stroma vs glandular epithelium ( $r=0.7, P<.0001$, Spearman's rank test) and glandular vs luminal epithelium ( $r=0.7, P<.0001$ ). Nevertheless, proportionally many more anillin $^{+}$cells reside in the stroma when compared with glandular or luminal epithelium (Figure S2).

We reasoned that the time-dependent increase in anillin ${ }^{+}$cells in the stroma could be accounted for by accumulation of proliferating uNK cells or macrophages prior to menstrual shedding. ${ }^{15}$ To test this conjecture, 21 endometrial biopsies were subjected to double-labeling immunofluorescence microscopy to quantify co-expression of anillin with CD163 or CD56, macrophage and uNK cell markers, respectively. ${ }^{6,36}$ To assess the contribution of proliferating leukocyte and EC precursors, the abundance of anillin ${ }^{+}$cells co-expressing CD34 was also quantified (Figure S3). ${ }^{37}$ The samples were selected to coincide with the implantation window $(\mathrm{LH}+7$ and $\mathrm{LH}+9)$ or the late-secretory phase of the cycle $(\mathrm{LH}+11)$.
Anillin ${ }^{+} \mathrm{CD} 6^{+}$cells were noticeably absent in all but one sample at $\mathrm{LH}+7$ but their abundance increased markedly upon transition to the late-secretory phase in parallel with anillin ${ }^{+} \mathrm{CD} 163^{+}$macrophages (Figure 2D). The reverse pattern was observed for anillin ${ }^{+} \mathrm{CD} 34^{+}$cells. At the start of the implantation window $(\mathrm{LH}+7), 61 \%$ of anillin ${ }^{+}$cells did not express IC markers, but this dropped to $32 \%$ by the late-secretory phase of the cycle (Figure 2E).

\section{3 | hPMC confer endometrial clonogenicity}

To determine if hPMC confer endometrial clonogenicity, we first established paired colony forming unit (CFU) assays and standard primary EnSC cultures from three biopsies (Figure 3A). After 7 days, the abundance of anillin ${ }^{+}$cells [median (IQR)] in standard cultures was 1.7 (1.9)\% vs 17 (7.7)\% in clonal assays $(P<.05$, Mann Whitney $U$ test). To test whether culturing leads to loss of proliferative capacity, anillin ${ }^{+}$cells were quantified using immunofluorescence microscopy in CFU assays after 3, 6, or 9 days in culture (Figure 3B). Notably, the abundance of anillin $^{+}$clonal cells on day 3 was 68 (17.7)\% but this dropped significantly by day 9 to 15.1 (9.6)\% ( $P<.0002$, Kruskal-Wallis test). Next, we examined if the abundance of anillin ${ }^{+}$cells in vivo is a measure of endometrial clonogenicity. CFU assays were established from 47 midluteal endometrial biopsies and, in parallel, anillin ${ }^{+}$cells were quantified in the stromal compartment using immunohistochemistry (Figure $3 \mathrm{C}$ ). A significant correlation was observed between the percentage of anillin ${ }^{+}$stromal cells in vivo and CFU activity of freshly isolated EnSC in vitro $(r=0.49$, $P<.0004$, Spearman's rank test)

\section{4 | Transcriptomic profiling of cultured clonogenic and resident stromal cells}

Previous studies reported that CFU assays are more efficient when established from SUSD2 ${ }^{+}$perivascular cell (PVC) fraction when
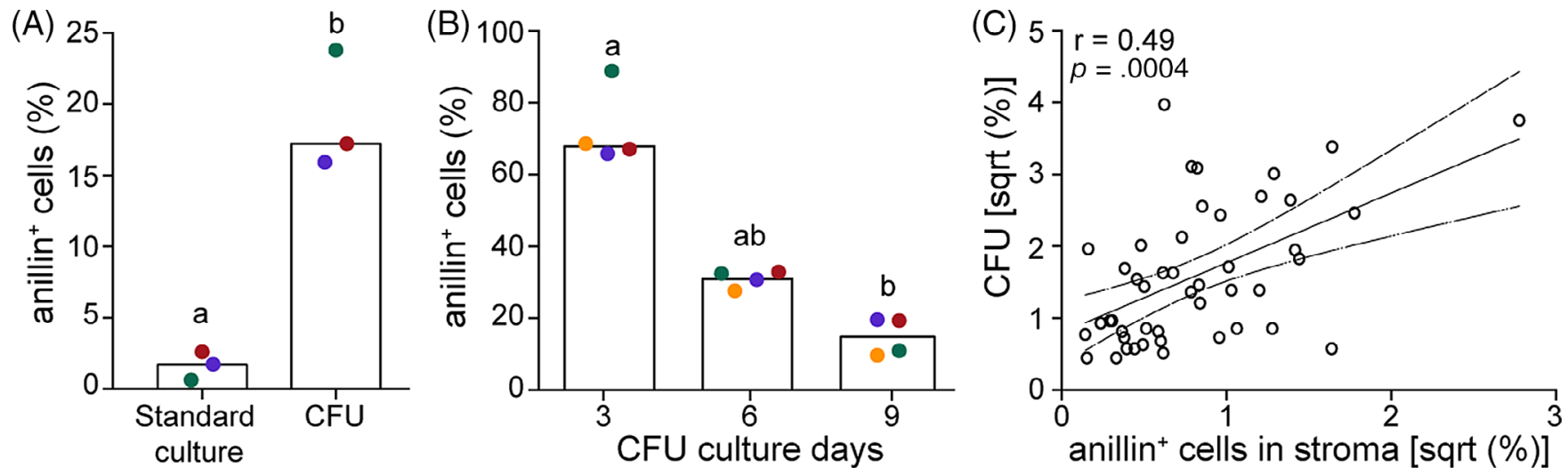

FIGURE 3 hPMC confer clonogenicity to midluteal superficial endometrium. A, Percentage of anillin ${ }^{+}$cells in primary EnSC cultures and CFU assays. Bar graphs show median of three biological repeat experiments. B, Percentage of anillin ${ }^{+}$cells in CFU assays on day 3,6 , and 9 of culture. Bar graphs show median of four biological repeat experiments. Different letters above the box plots indicate that groups are significantly different from each other at $P<.05$ (one-way ANOVA on ranks test). C, Spearman's rank correlation of anillin ${ }^{+}$cells in the endometrial stromal compartment in vivo and CFU activity of freshly isolated EnSC in 47 paired midluteal endometrial biopsies 
(A) endometrial
biopsy

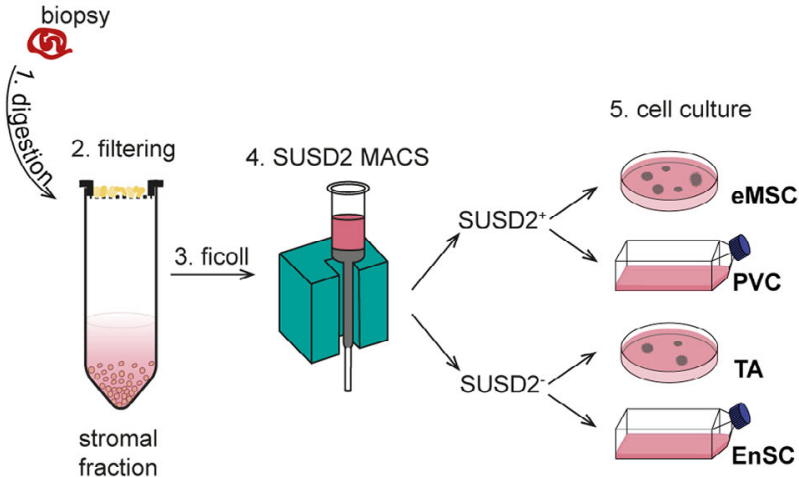

(B)

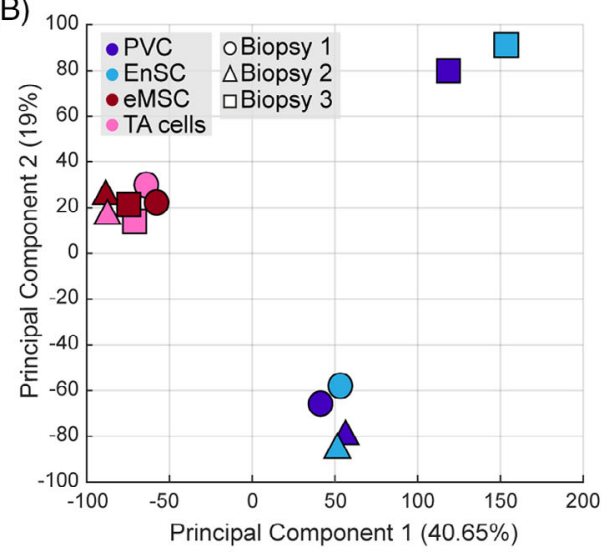

(C)
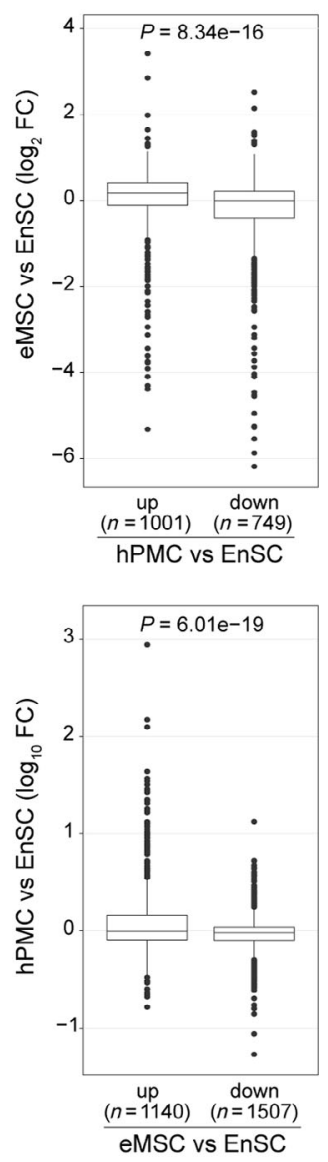

(D)

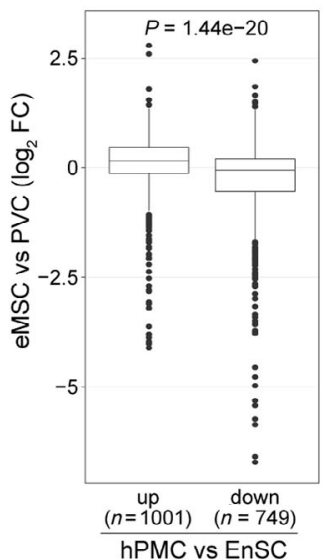

In vivo
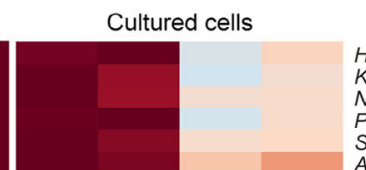

HJURP

KIF $4 A$

PBK

SHCBP1

ANLN

NUSAP1

DIAPH3

TOP2A

KIF23

KIF23

CKAP2L

MELK

SGOL1
CDC2O

ASPM

CD109

THSD 4

FBXL13

ALDH1L

ALDT

RAC2

GALNT

ANO4

MMP16

PFN2

UCHL1

HERC 3
PCDH9

LAG3

COL6A3

COL6A3
ATP2B1

ATP2B1

SKP2

PHLDA

ETV1

FOXF1

ITGA8

ELTD1

LRRC17

NCKAP5

COLEC

SLIT2

CXXC4

LYPD1

TRPV2

SOX9

EC EnSC hPMC eMSC TA cells EnSC PVC

FIGURE 4 A shared gene signature between clonogenic cells in culture and hPMC in vivo. A, Schematic depiction of the experimental design. After 10 days in culture, total RNA from three biological repeat experiments was subjected to bulk RNA-sequencing. B, Principal-component analysis of RNA-seq data of cultured clonogenic cells (eMSC/TA) and resident cells (EnSC/PVC). C, Upper panels: box plots showing relative expression in eMSC vs EnSC or eMSC vs PVC of genes either enriched or depleted in hPMC when compared with EnSC. Lower panel: box plot showing relative expression in hPMC vs EnSC of genes either significantly enriched or depleted in eMSC when compared with EnSC and PVC. For in vitro bulk RNA-seq data, DEGs were defined as $>2$-fold-change (FC) and FDR corrected $P<.05$. For in vivo scRNA-seq data, DEGs were defined as $P<.05$ and expression in $>10 \%$ of cells. D, Composite heatmap showing relative expression of indicated genes in EC, EnSC and hPMC in vivo and eMSC, TA cells, EnSC and PVC in vitro. The color key indicates the level of gene expression relative to the highest expressing cell population in vivo or in vitro 
(A) anillin $^{+}$CD163 $3^{+}$

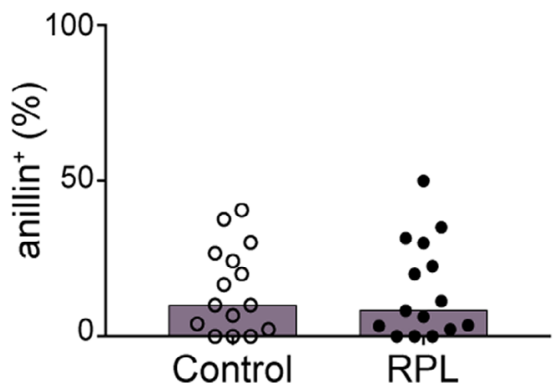

(B)

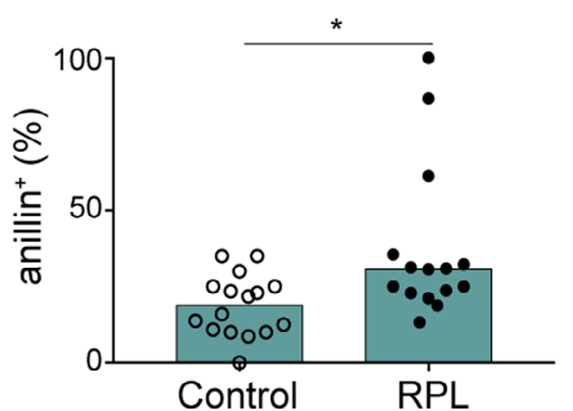

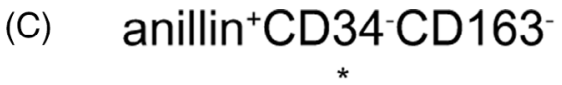

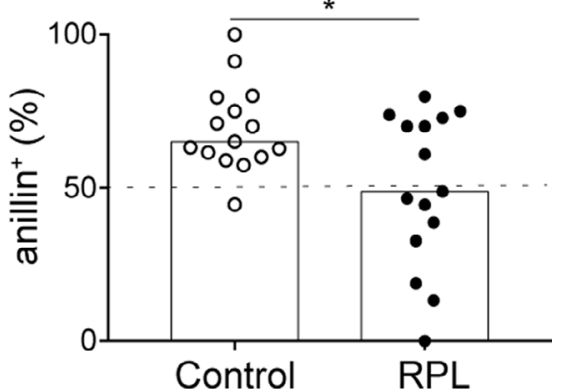

FIGURE 5 Loss of hPMC in recurrent pregnancy loss (RPL). The percentage of anillin ${ }^{+}$cells co-expressing, A, CD163 (macrophages) or, B, CD34 (hematopoietic/endothelial precursor cells) was quantified by immunofluorescent microscopy in endometrial biopsies obtained on LH +7 from control subjects $(n=15)$ and RPL patients $(n=15)$. C, The relative abundance of hPMC (ie, anillin ${ }^{+}$cells not co-expressing CD34 or CD163) in endometrial biopsies from control and RPL subjects. ${ }^{*}$ indicates $P<.05$ (Mann-Whitney $U$ test)

compared with non-perivascular (SUSD2-) EnSC. ${ }^{22,38}$ We reasoned that if hPMC are derived from circulating progenitor cells and account for endometrial clonicity during the implantation window, the transcriptomic profiles of clonogenic cells in culture should exhibit less variability when compared with cultured resident PVC or EnSC that have been exposed to iterative menstrual cycles in vivo. To test this hypothesis, we used magnetic-activated cell sorting to isolate SUSD2 ${ }^{+}$PVC and SUSD2- EnSC from three different biopsies and subjected both cell fractions to standard cultures as well as CFU assays (Figure 4 A). For clarity, clonogenic cells established from PVC were termed endometrial MSC (eMSC) whereas the term "transit amplifying" (TA) cells was coined to clonal cells in the EnSC fraction. After 10 days in culture, total RNA was extracted and subjected to RNA-seq analysis. Principal component (PC) analysis separated standard EnSC and PVC cultures from eMSC and TA in PC1 (Figure 4B). Notably, all six libraries from clonal cells clustered tightly together. Although PVC and EnSC also clustered together, there was considerable divergence in their transcriptional profiles between samples (Figure 4B). Thus, in keeping with our hypothesis, cultured resident cells exhibit much greater interpatient transcriptional variability when compared with clonogenic cells.

Based on $>2$-fold-change in gene expression and FDR corrected $P<.05$, only two transcripts (STMN3 and ITGBL1) were differentially expressed between eMSC and TA cells, indicating shared origins. By contrast, 3460 genes were differentially expressed between clonogenic cells (eMSC plus TA cells) and resident stromal cells (PVC plus EnSC). Out of the 3460 genes, $81.8 \%$ were down-regulated in clonal cells. The heatmap in Figure S4A depicts the 10 most highly enriched or depleted genes in clonal cells when compared with EnSC and PVC. In keeping with the results of a previous study, ${ }^{38}$ a total of 216 differentially expressed genes (DEG) were identified between cultured PVC and EnSC. Interesting, some genes enriched in PVC vs EnSC appeared also higher expressed in eMSC and, to a lesser extent, in TA cells, although statistical significance was often not reached (Figure S4A). An UpSet plot was used to depict the intersections of DEG in different comparisons (Figure S4B).

Clonogenic cells rapidly undergo spontaneous differentiation and senescence in culture. ${ }^{39}$ Nevertheless, differences in gene expression between $\mathrm{hPMC}$ vs EnSC in vivo were partially maintained in eMSC when compared with EnSC ( $P=8.3 \times 10^{-16}, t$-test $)$ or PVC $\left(P=1.4 \times 10^{-22}\right)$ in vitro (Figure $4 \mathrm{C}$ and Table S4). Conversely, differences in gene expression between clonal and resident cells in vitro were also partially maintained between hPMC and EnSC in vivo $\left(P=6.1 \times 10^{-19}\right)$ (Figure $\left.4 C\right)$. Mining of the data provided further evidence that hPMC are the progenitors of colony-forming cells in culture. As anticipated, proliferation-associated genes were higher expressed in eMSC/TA cells when compared with cultured EnSC or PVC (Figure 4D), including multiple genes implicated in cancer stem cells (eg, PBK, NUSAP1, MELK, CDC20, and ASPM). ${ }^{40-43}$ Furthermore, several MSC-related genes enriched in hPMC were also selectively enriched in eMSC/TA cells. For example, ALDH1L1, encoding aldehyde dehydrogenase 1 family member L1, and CD109 are putative biomarkers of cancer stem-like cells, ${ }^{44,45}$ whereas profilin 2 (PFN2) promotes migration, invasion and stemness of HT29 human colorectal cancer stem cells. ${ }^{46}$ Matrix metallopeptidase 16 (MMP16) controls the migration of human cardiomyocyte progenitor cells. ${ }^{47}$ Notably, eMSC, and to a lesser extent TA cells, also express an EC-like gene signature, albeit devoid of canonical endothelial marker genes (Figure 4D). Multiple genes in this signature, such as SKP2, ITGA8, SLIT2, and SOX9, have been shown to be implicated in different stem/progenitor cell niches. ${ }^{48-51}$

\subsection{Loss of hPMC in recurrent pregnancy loss}

We reported previously that recurrent pregnancy loss, defined here as three or more miscarriages, is associated with loss of eMSC and TA cells, as assessed by CFU assays on freshly isolated PVC and EnSC, respectively, from midluteal biopsies. ${ }^{14}$ Hence, we examined if this prevalent reproductive disorder is also associated with loss of anillin ${ }^{+}$ hPMC in vivo. To mitigate against interference from anillin ${ }^{+} \mathrm{IC}$, the analysis was restricted to endometrial biopsies obtained on $\mathrm{LH}+7$, a timepoint when anillin ${ }^{+} \mathrm{CD} 56^{+}$cells are virtually absent (Figure $2 \mathrm{D}$ ). A total of 30 biopsies from RPL patients $(n=15)$ and control subjects $(n=15)$ were processed for immunofluorescence microscopy to 
(A)
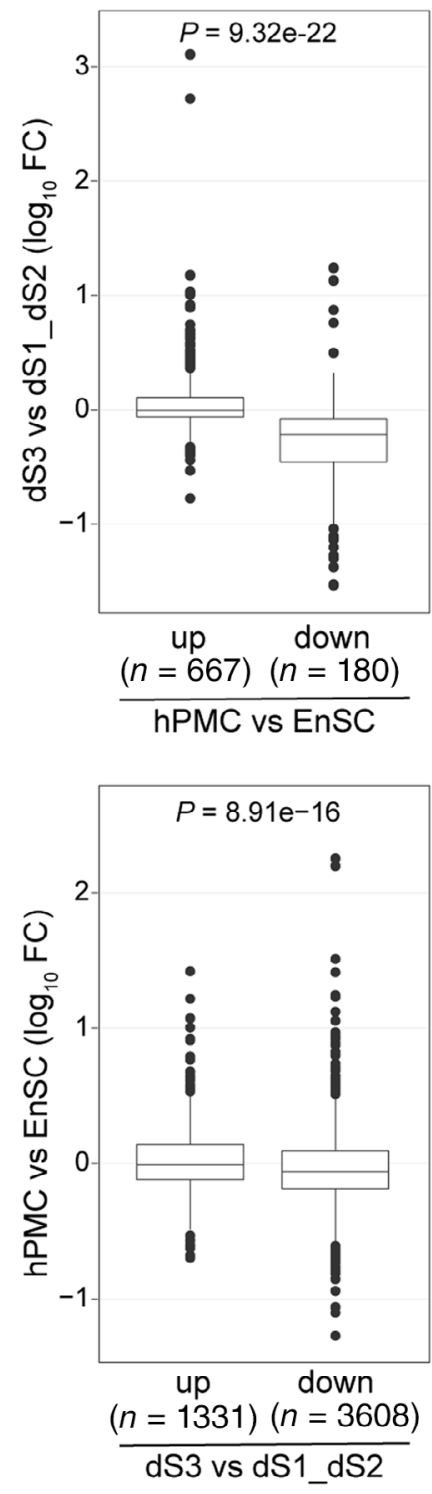

(B)

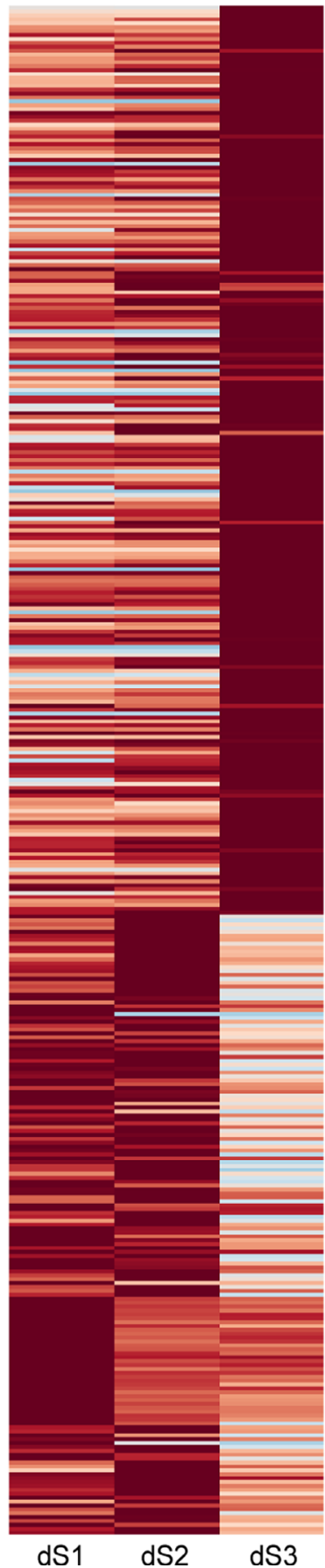

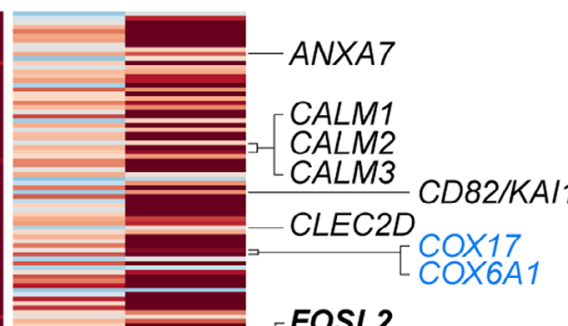

FOSL2

GAPDH

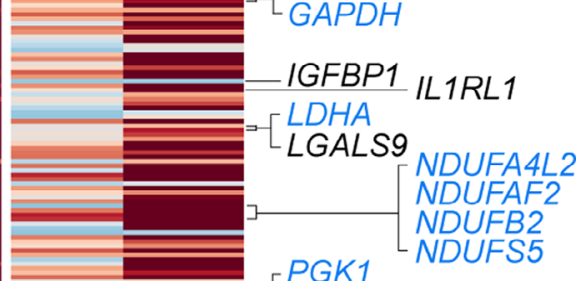

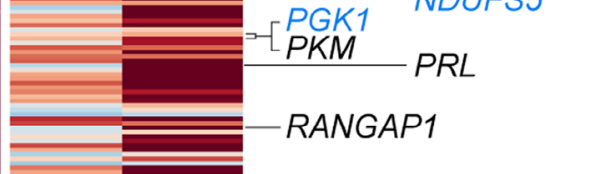
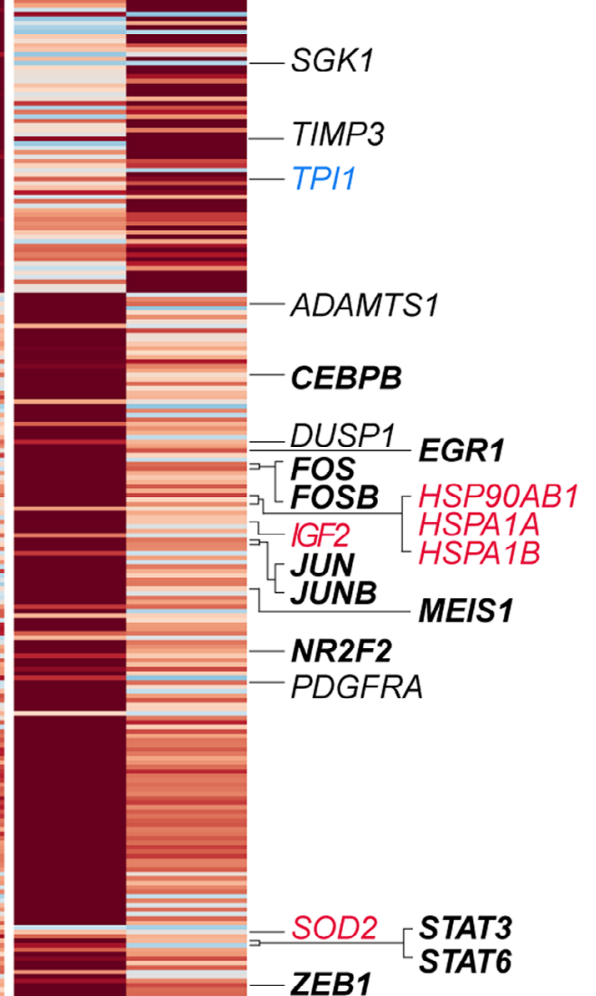

EnSC hPMC

FIGURE 6 hPMC are putative precursors of a distinct decidual subpopulation in pregnancy. A, Upper panel: box plots showing the relative expression in dS3 vs dS1 and dS2 subpopulations of genes either significantly $(P<.05$ and expression in $>10 \%$ of cells) enriched or depleted in hPMC when compared with EnSC and EC in midluteal endometrial. Lower panel: box plots showing the relative expression in hPMC vs EnSC of genes either significantly enriched or depleted in dS3 cells when compared with dS1 and dS2 populations at the maternal-fetal interface in early pregnancy. B, Composite heatmap showing relative expression of indicated genes in the three decidual subsets (dS1-3) at the maternal-fetal interface in early gestation and in EnSC and hPMC during the window of implantation. Annotated genes were color-coded to indicate stress-related genes (red), mitochondrial and metabolic genes (blue), or genes coding for known decidual factors (black) or transcription factors (bold black). The color key indicates the level of gene expression relative to the highest expressing cell population either in pregnancy (dS1-3) or during the implantation window prior to conception (EnSC and hPMC)

quantify anillin ${ }^{+}$cells in the stromal compartment as well anillin ${ }^{+}$cells co-expressing CD163 or CD34. While the percentage of ani$\mathrm{Ilin}^{+} \mathrm{CD} 163^{+}$cells did not differ between the two clinical groups
(Figure 5A), a significant increase in anillin ${ }^{+} \mathrm{CD} 34^{+}$cells was observed in RPL patients when compared with control subjects $(P<.05$, MannWhitney test; Figure 5B). Conversely, RPL was associated with a 
reciprocal reduction in $\mathrm{hPMC}$, that is, anillin ${ }^{+}$cells that do not express IC markers ( $P<.05$, Mann-Whitney $U$ test; Figure $5 C$ ). The abundance of $\mathrm{hPMC}$ population was noticeably more variable in tissue samples from RPL patients when compared with control subjects (Figure $5 \mathrm{C}$ ). Furthermore, hPMC made up $>50 \%$ of all anillin ${ }^{+}$cells in 14 out of 15 control samples compared with only 7 out of 15 samples in the RPL group ( $P=.01$, Fisher's exact test).

\section{6 | hPMC map to a distinct decidual subpopulation in pregnancy}

Next, we set out to determine the potential fate of hPMC in early pregnancy. To do this, we made use of the recently published singlecell atlas of the maternal-fetal interface in early gestation. ${ }^{13}$ Anatomically, the maternal decidua consists of two distinct layers, the decidua compacta near the surface epithelium and the underlying decidua spongiosa, which contains hypersecretory glands. The two decidual layers contain three decidual subpopulations, designated dS1-3 (Figure S5A). ${ }^{13}$ The decidua spongiosa harbors dS1 cells expressing ACTA2 whereas the dS2 and dS3 cells in the decidua compacta share the expression of DCN (Figure S5B). Notably, dS3 cells constitute a minor subpopulation, representing only $7.2 \%$ of all decidual stromal cells at the maternal-fetal interface. Computational analysis indicated that $7 \%$ of dS3 cells are in S or G2/M phase of the cell cycle compared with $2.6 \%$ and $3.1 \%$ of dS2 and dS1 cells, respectively. Although dS3 cells are much less proliferative than hPMC, they nevertheless share a conspicuous gene signature (Figure 6A and Table S5). This signature is not only enriched in several canonical decidual genes, including PRL, IL1RL1, and CD82 (also known as $K A I 1$ ), ${ }^{7,25,52}$ but also in genes involved in glycolysis and mitochondrial function (Figure 6B and Figure S5B). Conversely, hPMC in midluteal endometrium and dS3 cells at the maternal-fetal interface in pregnancy are relatively depleted in genes implicated in cellular stress responses (Figure 6B), including members of the AP-1 family of transcription factors (FOS, FOSB, JUN, and JUNB) and CEBP $\beta$ (CEBPB), which effects free radical signaling in decidual cells. ${ }^{53}$ Based on this shared metabolic and decidual gene signature, we conclude that hPMC likely give rise in pregnancy to a distinct decidual subpopulation that engages with invading placental trophoblast in the decidua compacta.

\section{4 | DISCUSSION}

Decidual transformation of human endometrium upon embryo implantation requires differentiation of resident EnSC, immune clearance of acute senescent decidual cells, and rapid tissue growth. These processes occur in concert with coordinated trophoblast invasion. ${ }^{54}$ Considering the magnitude of tissue remodeling required for pregnancy, poised progenitor and highly proliferative decidual precursor cells are likely critical for the formation of a robust maternal-fetal interface, as is the case in mice. ${ }^{20}$ Here, we provide evidence of recruitment, engraftment, and differentiation of hPMC/decidual precursor cells during the window of implantation in human endometrium. Transcriptional profiling highlighted the transitional nature of hPMC, exemplified on the one hand by expression of genes involved in chemotaxis, EC interactions, migration, and engraftment and, on the other, by expression of decidual transcription factors and marker genes. Human BDMSC are capable of differentiating into prolactinproducing decidual cells in vitro, ${ }^{55}$ further supporting our assertion that hPMC are equivalent to bone marrow-derived decidual precursor cells in pregnant mice. We demonstrate that hPMC share a conspicuous gene signature with dS3 cells identified recently in the decidua compacta of the maternal-fetal interface. ${ }^{13}$ This common gene signature is defined by the expression of specific decidual marker genes, including PRL, IL1RL1, and CD82,7,25,52 enhanced expression of mitochondrial and metabolic genes, and the relative lack of stress-related genes when compared with either resident EnSC in midluteal endometrium or other decidual subsets present at the maternal-fetal interface in pregnancy. Furthermore, a hallmark of dS3 cells in pregnancy is the expression of $H S D 11 B 1,{ }^{13}$ which converts inactive cortisone into cortisol, a potent anti-inflammatory glucocorticoid. ${ }^{56}$ Although our computational analysis showed that the proliferative capacity of dS3 cells is higher than that of other decidual subsets, it is markedly lower when compared with hPMC. It is plausible that recruitment of hPMC to the maternal-fetal interface diminishes rapidly upon invasion and plugging of the maternal terminal arterioles by extravillous trophoblast and subsequent physiological transformation of the spiral arteries. ${ }^{57-59}$ If correct, the level of cellular plasticity in the decidual basalis, and thus protection against decidual senescence throughout gestation, may be determined during a relatively narrow window in early gestation.

Studies on the role of BDMSC in endometrial physiology have largely focused on tissue regeneration. ${ }^{60-62}$ Menstrual "injury" and rising estradiol levels upregulate $\mathrm{CXCL12}$, a potent chemotactic factor that mediates mobilization and homing of BDMSC by activating CXCR4. ${ }^{63}$ It is unclear whether the CXCL12/CXCR4 axis also mediates recruitment of BDMSC during the progesterone-dominant luteal phase. Notably, the expression of the evolutionarily related chemokine CXCL14 is at least a magnitude higher during the window of implantation when compared with CXCL12.,8 CXCL14 is an orphan chemokine acting on uNK cells and immature dendritic cells but not $\mathrm{T}$ cells. ${ }^{64}$ It also stimulates fibroblast proliferation and migration, ${ }^{65}$ as well as embryonic stem cell renewal. ${ }^{32}$ The peri-implantation endometrium is further characterized by increased vascular permeability, ${ }^{66}$ which arguably facilitates extravasation of circulating hematopoietic and nonhematopoietic cells. BDMSC also give rise to EC progenitor cells in both human and murine endometrium. ${ }^{20,62,67,68}$ This shared ontogeny with hPMC may account for our observation that clonogenic cells in culture spontaneously express EC-related genes.

Our characterization of bone marrow-derived decidual precursors in human endometrium has potentially far-reaching clinical implications. As mentioned, bone marrow transplantation experiments in mice provided elegant proof-of-concept evidence of the therapeutic potential of BDMSC, demonstrating that wild-type BDMSC restore uterine defects, enhance decidualization, and prevent pregnancy loss 
in Hoxa10 $0^{+/-}$mice. ${ }^{20}$ We reported previously that recurrent pregnancy loss is associated with loss of endometrial clonogenicity in midluteal endometrium, measured by CFU assays on freshly isolated $\mathrm{EnSC},{ }^{14}$ and increased frequency of menstrual cycles with a pro-senescent decidual response. ${ }^{8}$ We now demonstrate that a lack of decidual precursor cells accounts for the loss of endometrial clonogenicity in recurrent miscarriage. A history of miscarriage also increases the risk of preterm birth, ${ }^{69,70}$ the leading cause of neonatal death and morbidity worldwide. ${ }^{71}$ Interestingly, while recurrent pregnancy loss is associated with an overt pro-senescent decidual response, ${ }^{8,16}$ spontaneous preterm labor is linked to accelerated decidual aging and loss of plasticity at the maternal-fetal interface. ${ }^{72,73}$ Hence, it is plausible that treatments aimed at enhancing recruitment, engraftment and differentiation of hPMC in the endometrium prior to, or soon after, conception will not only reduce the risk of miscarriage but also prevent preterm labor in at risk women. In this context, a recent pilot trial demonstrated that sitagliptin, a dipeptidyl-peptidase IV inhibitor used in the management of diabetes, increases endometrial clonogenicity and decreases decidual senescence during the implantation window in recurrent miscarriage patients. ${ }^{74}$ The efficacy of sitagliptin, and other potential interventions that target BDMSC and decidual precursor cells, ${ }^{28}$ in mitigating the risk of adverse pregnancy outcome warrants further evaluation in clinical trials.

\section{5 | CONCLUSION}

This study profiles the transcriptome of decidual precursor cells during the window of implantation in human endometrium. Based on our findings, we posit that decidual precursors are derived from circulating BDMSC, primed for exponential growth in early pregnancy, and integral to the formation of the decidua compacta of the maternal-fetal interface. Conversely, lack of decidual precursors in a conception cycle may trigger a concatenation of events, resulting in breakdown of the decidua in early gestation and miscarriage or, plausibly, accelerated aging of the maternal-fetal interface and spontaneous preterm labor.

\section{ACKNOWLEDGMENTS}

We are grateful to all the women who participated in this research and those who facilitated their participation. We also thank the HighThroughput Genomics Group at the Wellcome Trust Centre for Human Genetics for the generation of sequencing data. This work was supported by funds from the Tommy's National Miscarriage Research Centre and Wellcome Trust Investigator Award to J.J.B. (212233/Z/18/Z).

\section{CONFLICT OF INTEREST}

The authors declared no potential conflicts of interest.

\section{AUTHOR CONTRIBUTIONS}

M.D., C.K., K.J.F., P.J.B., S.O., E.S.L., P.V.: collection and/or assembly of data, data analysis and interpretation, manuscript writing, final approval of manuscript; T.R.: data analysis and interpretation, manuscript writing, final approval of manuscript; A.H., J.O., S.Q.: provision of study material or patients, data analysis and interpretation, final approval of manuscript; J.J.B.: conception and design, provision of study material or patients, collection and/or assembly of data, data analysis and interpretation, manuscript writing, final approval of manuscript.

\section{DATA AVAILABILITY STATEMENT}

RNA-seq data were deposited in the Gene Expression Omnibus (http://www.ncbi.nlm.nih.gov/geo/), accession number GSE159266.

\section{ORCID}

Jan J. Brosens (D) https://orcid.org/0000-0003-0116-9329

\section{REFERENCES}

1. Gargett CE, Schwab KE, Deane JA. Endometrial stem/progenitor cells: the first 10 years. Hum Reprod Update. 2016;22(2):137-163.

2. Moore L, Leongamornlert $\mathrm{D}$, Coorens $\mathrm{THH}$, et al. The mutational landscape of normal human endometrial epithelium. Nature. 2020;580 (7805):640-646.

3. Gellersen B, Brosens JJ. Cyclic decidualization of the human endometrium in reproductive health and failure. Endocr Rev. 2014;35(6): 851-905.

4. Wang W, Vilella F, Alama $P$, et al. Single-cell transcriptomic atlas of the human endometrium during the menstrual cycle. Nat Med. 2020 26(10):1644-1653

5. Erkenbrack EM, Maziarz JD, Griffith OW, et al. The mammalian decidual cell evolved from a cellular stress response. PLoS Biol. 2018;16(8): e2005594.

6. Brighton PJ, Maruyama Y, Fishwick K, et al. Clearance of senescent decidual cells by uterine natural killer cells in cycling human endometrium. Elife. 2017;6:e31274. https://doi.org/10.7554/eLife.31274

7. Salker MS, Nautiyal J, Steel JH, et al. Disordered IL-33/ST2 activation in decidualizing stromal cells prolongs uterine receptivity in women with recurrent pregnancy loss. PLoS One. 2012;7(12):e52252.

8. Lucas ES, Vrljicak P, Muter J, et al. Recurrent pregnancy loss is associated with a pro-senescent decidual response during the periimplantation window. Commun Biol. 2020;3(1):37.

9. Kajihara T, Jones M, Fusi L, et al. Differential expression of FOXO1 and FOXO3a confers resistance to oxidative cell death upon endometrial decidualization. Mol Endocrinol. 2006;20(10):2444-2455.

10. Muter J, Alam MT, Vrljicak P, et al. The Glycosyltransferase EOGT regulates Adropin expression in decidualizing human endometrium. Endocrinology. 2018;159(2):994-1004.

11. Muter J, Brighton PJ, Lucas ES, et al. Progesterone-dependent induction of phospholipase C-related catalytically inactive protein 1 (PRIP-1) in decidualizing human endometrial stromal cells. Endocrinology. 2016; 157(7):2883-2893.

12. Muter J, Lucas ES, Chan YW, et al. The clock protein period 2 synchronizes mitotic expansion and decidual transformation of human endometrial stromal cells. FASEB J. 2015;29(4):1603-1614.

13. Vento-Tormo R, Efremova $M$, Botting RA, et al. Single-cell reconstruction of the early maternal-fetal interface in humans. Nature. 2018;563(7731):347-353.

14. Lucas ES, Dyer NP, Murakami K, et al. Loss of endometrial plasticity in recurrent pregnancy loss. STEM CELLS. 2016;34(2):346-356.

15. Jabbour HN, Kelly RW, Fraser HM, Critchley HOD. Endocrine regulation of menstruation. Endocr Rev. 2006;27(1):17-46.

16. Ewington LJ, Tewary S, Brosens JJ. New insights into the mechanisms underlying recurrent pregnancy loss. J Obstet Gynaecol Res. 2019; 45(2):258-265 
17. Ticconi C, Pietropolli A, D'lppolito S, et al. Time-to-pregnancy in women with unexplained recurrent pregnancy loss: a controlled study. Reprod Sci. 2020;27(5):1121-1128.

18. Kearns M, Lala PK. Bone marrow origin of decidual cell precursors in the pseudopregnant mouse uterus. J Exp Med. 1982;155(5):15371554.

19. Lysiak JJ, Lala PK. In situ localization and characterization of bone marrow-derived cells in the decidua of normal murine pregnancy. Biol Reprod. 1992;47(4):603-613.

20. Tal R, Shaikh S, Pallavi $P$, et al. Adult bone marrow progenitors become decidual cells and contribute to embryo implantation and pregnancy. PLoS Biol. 2019;17(9):e3000421.

21. Barros FSV, Brosens JJ, Brighton PJ. Isolation and primary culture of various cell types from whole human endometrial biopsies. Bio-Protocol. 2016;6(22).

22. Murakami K, Bhandari H, Lucas ES, et al. Deficiency in clonogenic endometrial mesenchymal stem cells in obese women with reproductive failure-a pilot study. PLoS One. 2013;8(12):e82582.

23. Tirosh I, Izar B, Prakadan SM, et al. Dissecting the multicellular ecosystem of metastatic melanoma by single-cell RNA-seq. Science. 2016;352(6282):189-196.

24. Efremova M, Vento-Tormo M, Teichmann SA, Vento-Tormo $R$ CellPhoneDB: inferring cell-cell communication from combined expression of multi-subunit ligand-receptor complexes. Nat Protoc. 2020;15(4):1484-1506.

25. Brosens JJ, Hayashi N, White JO. Progesterone receptor regulates decidual prolactin expression in differentiating human endometrial stromal cells. Endocrinology. 1999;140(10):4809-4820.

26. Huyen DV, Bany BM. Evidence for a conserved function of heart and neural crest derivatives expressed transcript 2 in mouse and human decidualization. Reproduction. 2011;142(2):353-368.

27. Lynch VJ, Brayer K, Gellersen B, Wagner GP. HoxA-11 and FOXO1A cooperate to regulate decidual prolactin expression: towards inferring the core transcriptional regulators of decidual genes. PLoS One. 2009; 4(9):e6845.

28. Liesveld JL, Sharma N, Aljitawi OS. Stem cell homing: from physiology to therapeutics. STEM CELLS. 2020;38(10):1241-1253.

29. Yeo GC, Weiss AS. Soluble matrix protein is a potent modulator of mesenchymal stem cell performance. Proc Natl Acad Sci USA. 2019; 116(6):2042-2051.

30. Himburg HA, Termini CM, Schlussel L, et al. Distinct bone marrow sources of Pleiotrophin control hematopoietic stem cell maintenance and regeneration. Cell Stem Cell. 2018;23(3):370-381. e375.

31. Gupta R, Turati V, Brian D, et al. Nov/CCN3 enhances cord blood engraftment by rapidly recruiting latent human stem cell activity. Cell Stem Cell. 2020;26(4):527-541. e528.

32. Cheng CL, Yang SC, Lai CY, et al. CXCL14 maintains hESC self-renewa through binding to IGF-1R and activation of the IGF-1R pathway. Cells. 2020;9(7):1706. https://doi.org/10.3390/cells9071706

33. Mokhtar NM, Cheng CW, Cook E, Bielby H, Smith SK, Charnock-Jones DS. Progestin regulates chemokine (C-X-C motif) ligand 14 transcript level in human endometrium. Mol Hum Reprod. 2010;16(3):170-177.

34. Uhlen M, Fagerberg L, Hallstrom BM, et al. Proteomics. Tissue-based map of the human proteome. Science. 2015;347(6220):1260419.

35. Zhang L, Maddox AS. Anillin. Curr Biol. 2010;20(4):R135-R136.

36. Fabriek BO, Dijkstra CD, van den Berg TK. The macrophage scavenger receptor CD163. Immunobiology. 2005;210(2-4):153-160.

37. Berenson RJ, Andrews RG, Bensinger WI, et al. Antigen CD34+ marrow cells engraft lethally irradiated baboons. J Clin Invest. 1988; 81(3):951-955.

38. Murakami K, Lee $\mathrm{YH}$, Lucas ES, et al. Decidualization induces a secretome switch in perivascular niche cells of the human endometrium. Endocrinology. 2014;155(11):4542-4553.

39. Lucciola R, Vrljicak P, Gurung S, et al. Impact of sustained transforming growth factor-beta receptor inhibition on chromatin accessibility and gene expression in cultured human endometrial MSC. Front Cell Dev Biol. 2020;8:567610.

40. Bikeye SN, Colin C, Marie Y, et al. ASPM-associated stem cell proliferation is involved in malignant progression of gliomas and constitutes an attractive therapeutic target. Cancer Cell Int. 2010;10:1.

41. Dougherty JD, Garcia AD, Nakano I, et al. PBK/TOPK, a proliferating neural progenitor-specific mitogen-activated protein kinase kinase. J Neurosci. 2005;25(46):10773-10785.

42. Li H, Zhang W, Yan M, et al. Nucleolar and spindle associated protein 1 promotes metastasis of cervical carcinoma cells by activating Wnt/beta-catenin signaling. J Exp Clin Cancer Res. 2019;38(1):33.

43. Ren L, Deng B, Saloura V, Park JH, Nakamura Y. MELK inhibition targets cancer stem cells through downregulation of SOX2 expression in head and neck cancer cells. Oncol Rep. 2019;41(4):2540-2548.

44. Douville J, Beaulieu R, Balicki D. ALDH1 as a functional marker of cancer stem and progenitor cells. Stem Cells Dev. 2009;18(1):17-25.

45. Emori M, Tsukahara T, Murase M, et al. High expression of CD109 antigen regulates the phenotype of cancer stem-like cells/cancerinitiating cells in the novel epithelioid sarcoma cell line ESX and is related to poor prognosis of soft tissue sarcoma. PLoS One. 2013;8 (12):e84187.

46. Kim MJ, Lee YS, Han GY, Lee HN, Ahn C, Kim CW. Profilin 2 promotes migration, invasion, and stemness of HT29 human colorectal cancer stem cells. Biosci Biotechnol Biochem. 2015;79(9):1438-1446.

47. Liu J, van Mil A, Aguor EN, et al. MiR-155 inhibits cell migration of human cardiomyocyte progenitor cells (hCMPCs) via targeting of MMP-16. J Cell Mol Med. 2012;16(10):2379-2386.

48. Kadaja M, Keyes BE, Lin M, et al. SOX9: a stem cell transcriptional regulator of secreted niche signaling factors. Genes Dev. 2014;28(4): 328-341.

49. Polisetti N, Zenkel M, Menzel-Severing J, Kruse FE, SchlötzerSchrehardt $U$. Cell adhesion molecules and stem cell-nicheinteractions in the Limbal stem cell niche. STEM CELLS. 2016;34(1): 203-219.

50. Shibata F, Goto-Koshino Y, Morikawa Y, et al. Roundabout 4 is expressed on hematopoietic stem cells and potentially involved in the niche-mediated regulation of the side population phenotype. STEM CELLS. 2009;27(1):183-190.

51. Wang J, Huang Y, Guan Z, et al. E3-ligase Skp2 predicts poor prognosis and maintains cancer stem cell pool in nasopharyngeal carcinoma. Oncotarget. 2014;5(14):5591-5601.

52. Gellersen B, Briese J, Oberndorfer M, et al. Expression of the metastasis suppressor KAl1 in decidual cells at the human maternal-fetal interface: regulation and functional implications. Am J Pathol. 2007; 170(1):126-139.

53. Al-Sabbagh M, Fusi L, Higham J, et al. NADPH oxidase-derived reactive oxygen species mediate decidualization of human endometrial stromal cells in response to cyclic AMP signaling. Endocrinology. 2011; 152(2):730-740.

54. Aplin JD, Myers JE, Timms K, Westwood M. Tracking placental development in health and disease. Nat Rev Endocrinol. 2020;16(9): 479-494.

55. Aghajanova L, Horcajadas JA, Esteban FJ, Giudice LC. The bone marrow-derived human mesenchymal stem cell: potential progenitor of the endometrial stromal fibroblast. Biol Reprod. 2010;82(6):10761087.

56. Kuroda K, Venkatakrishnan R, Salker MS, et al. Induction of 11betaHSD 1 and activation of distinct mineralocorticoid receptor- and glucocorticoid receptor-dependent gene networks in decidualizing human endometrial stromal cells. Mol Endocrinol. 2013;27(2):192-202.

57. Brosens I, Brosens JJ, Muter J, Puttemans P, Benagiano G. Preeclampsia: the role of persistent endothelial cells in uteroplacental arteries. Am J Obstet Gynecol. 2019;221(3):219-226.

58. Burton GJ, Jauniaux E. The cytotrophoblastic shell and complications of pregnancy. Placenta. 2017;60:134-139. 
59. Burton GJ, Jauniaux E, Watson AL. Maternal arterial connections to the placental intervillous space during the first trimester of human pregnancy: the Boyd collection revisited. Am J Obstet Gynecol. 1999; 181(3):718-724.

60. Du H, Taylor HS. Contribution of bone marrow-derived stem cells to endometrium and endometriosis. Stem Cells. 2007;25(8):2082-2086.

61. Sahin Ersoy G, Zolbin MM, Cosar E, Moridi I, Mamillapalli R, Taylor HS. CXCL12 promotes stem cell recruitment and uterine repair after injury in Asherman's syndrome. Mol Ther Methods Clin Dev. 2017;4:169-177.

62. Taylor HS. Endometrial cells derived from donor stem cells in bone marrow transplant recipients. JAMA. 2004;292(1):81-85.

63. Yi KW, Mamillapalli R, Sahin C, Song J, Tal R, Taylor HS. Bone marrow-derived cells or C-X-C motif chemokine 12 (CXCL12) treatment improve thin endometrium in a mouse model. Biol Reprod. 2019;100(1):61-70.

64. Lu J, Chatterjee M, Schmid H, Beck S, Gawaz M. CXCL14 as an emerging immune and inflammatory modulator. J Inflamm (Lond). 2016;13:1.

65. Augsten M, Hagglof C, Olsson E, et al. CXCL14 is an autocrine growth factor for fibroblasts and acts as a multi-modal stimulator of prostate tumor growth. Proc Natl Acad Sci USA. 2009;106(9):3414-3419.

66. Goddard LM, Murphy TJ, Org T, et al. Progesterone receptor in the vascular endothelium triggers physiological uterine permeability preimplantation. Cell. 2014;156(3):549-562.

67. Mints $M$, Jansson M, Sadeghi B, et al. Endometrial endothelial cells are derived from donor stem cells in a bone marrow transplant recipient. Hum Reprod. 2008;23(1):139-143.

68. Tal R, Dong D, Shaikh S, Mamillapalli R, Taylor HS. Bone-marrowderived endothelial progenitor cells contribute to vasculogenesis of pregnant mouse uterusdagger. Biol Reprod. 2019;100(5):1228-1237.

69. Buchmayer SM, Sparen P, Cnattingius S. Previous pregnancy loss: risks related to severity of preterm delivery. Am J Obstet Gynecol. 2004;191(4):1225-1231.
70. Weintraub AY, Sergienko R, Harlev A, et al. An initial miscarriage is associated with adverse pregnancy outcomes in the following pregnancy. Am J Obstet Gynecol. 2011;205(3):286e281-286e285.

71. Blencowe $\mathrm{H}$, Cousens $\mathrm{S}$, Oestergaard $\mathrm{MZ}$, et al. National, regional, and worldwide estimates of preterm birth rates in the year 2010 with time trends since 1990 for selected countries: a systematic analysis and implications. Lancet. 2012;379(9832):2162-2172.

72. Deng W, Cha J, Yuan J, et al. p53 coordinates decidual sestrin 2/AMPK/mTORC1 signaling to govern parturition timing. J Clin Invest. 2016;126(8):2941-2954.

73. Wijaya JC, Khanabdali R, Georgiou HM, et al. Functional changes in decidual mesenchymal stem/stromal cells are associated with spontaneous onset of labour. Mol Hum Reprod. 2020;26(8):636-651.

74. Tewary S, Lucas ES, Fujihara R, et al. Impact of sitagliptin on endometrial mesenchymal stem-like progenitor cells: a randomised, double-blind placebo-controlled feasibility trial. EBioMedicine. 2020;51:102597.

\section{SUPPORTING INFORMATION}

Additional supporting information may be found online in the Supporting Information section at the end of this article.

How to cite this article: Diniz-da-Costa M, Kong C-S, Fishwick KJ, et al. Characterization of highly proliferative decidual precursor cells during the window of implantation in human endometrium. Stem Cells. 2021;1-14. https://doi.org/ 10.1002/stem.3367 